\title{
HEALTH ASSESSMENT OF THE AQUATIC ECOSYSTEM IN AIYI RIVER
}

\author{
YIN, L. ${ }^{1}-$ QIU, X. ${ }^{2 *}-$ ZHAO, R. ${ }^{2}$ \\ ${ }^{1}$ School of Civil and Conservancy Engineering, Ningxia University, Yinchuan, Ningxia 750021, \\ China \\ ${ }^{2}$ College of Life Science, Ningxia University, Yinchuan, Ningxia 750021, China \\ *Corresponding author \\ e-mail: yinliang1001@163.com; phone: +86-187-2133-4685 \\ (Received $9^{\text {th }}$ Apr 2019; accepted 19 ${ }^{\text {th }}$ Jun 2019)
}

\begin{abstract}
Based on the investigation of the water environment factors and aquatic biodiversity of AiYi River from March to November 2018, 14 indicators were selected from water environment factors and aquatic biodiversity index using principal component analysis to construct the evaluation index system of aquatic ecosystem health of AiYi River. The gray correlative degree method was also used to evaluate the aquatic ecosystem's health condition of AiYi River from March to November 2018. The result showed that the aquatic ecosystem health condition of AiYi River was healthy in March and November, and that was sub-health in May, July, September. The aquatic ecosystem health of AiYi River was in the subhealth state due to the higher content of nitrogen, phosphorus and organic matter.

Keywords: aquatic ecosystem, principal component analysis, gray correlative degree analysis
\end{abstract}

\section{Introduction}

Ecosystem health refers to the structural integrity and functional stability of the ecosystem, and the ability of self-maintenance and self-repair. A healthy aquatic ecosystem plays an important role in protecting the water environment and maintaining its normal functions of the water environment. Accurate diagnosis and evaluation of the health status of the aquatic ecosystem has high theoretical and practical significance.

Aquatic ecosystem is a complex large-scale system. It is difficult to diagnose and evaluate the health status of aquatic ecosystem accurately by a single method. At present, the widely used method in the health evaluation of aquatic ecosystem is the index system method (Hossain et al., 2017; Milani et al., 2017; Janssens et al., 2017; Rongrong et al., 2018). A reasonable index system should reflect not only the overall health level of aquatic ecosystems, but also the changing trend of aquatic ecosystem health. The "Clean Water Act" issued by the United States in 1972 holds that maintaining the physical, chemical and biological integrity of the structure and function of river aquatic ecosystems is an important principle for river health assessment (Milani et al., 2017). Obolewski et al. (2019) proposed 16 indicators in 2019, including riparian zone integrity, river bed conditions, fish, aquatic plants, and classified the river health into five grades for evaluation. Some work has been done on the evaluation index system and methods of aquatic ecosystem health in China. Yu et al. (2017) put forward the contents of aquatic ecosystem health evaluation, such as the quality evaluation of biological habitat, hydrological evaluation and the evaluation of biological community. Maloney (2019) put forward the evaluation index system of water quantity, water quality, aquatic organism, physical structure and riparian zone, 
as well as five evaluation criteria of "very healthy, healthy, sub-healthy, unhealthy and sick". Du et al. ( 2017) constructed the evaluation index system of aquatic ecosystem health, and used the analytic hierarchy process to evaluate the Luanhe River Basin comprehensively.

Although the index system method can well reflect the health status of aquatic ecosystems well, selecting reasonable indicators to construct the evaluation index system has not yet formed a recognized theoretical system and methods. In this study, the principal component analysis method was used to screen the candidate indicators for the health assessment of the Aquatic Ecosystem of Aiyi River, construct the evaluation index system, determine the weight of the corresponding indicators, and use the grey correlation method to evaluate the health status of the Aquatic Ecosystem of Aiyi River comprehensively, in order to provide some basis for the restoration of the Aquatic Ecosystem of Aiyi River and the comprehensive prevention and control of water pollution.

\section{Materials and methods}

\section{Research area}

Ningxia is short of water resources, arid and windy, and vegetation is scarce, which determines that its environmental capacity is small, the stability of ecosystem is poor, and the water environment is vulnerable to pollution and destruction. At present, the water environment situation in Ningxia is severe, and the regional water environmental pollution has become one of the biggest environmental problems. Aiyi River is the key project of river-lake system connection in Yinchuan City. It is the key water conservancy project of autonomous region integrating flood control, drainage, ecology and landscape. It is a National Water Conservancy Scenic spot. It has important social significance and far-reaching history for improving flood control and drainage conditions, Regulating Groundwater level, beautifying human settlement environment, upgrading urban grade, realizing human-water harmony and social harmony in Yinchuan City. Significance. The total length of Aiyi River is $158.5 \mathrm{~km}$, the water surface is more than $50,000 \mathrm{mu}$, and the drainage area is controlled at 1.75 million mu. The main source of water is flood, Gully Water and channel discarded water. The amount of water that can be reused in the upper reaches of Aiyi River reaches 51 million $\mathrm{m} 3$ every year, of which farmland drainage accounts for $68.9 \%$, rich in nitrogen and phosphorus nutrients. Agricultural and rural non-point source pollution in irrigation area is becoming the basic restrictive factor of the water quality of Aiyi River, and the backwater pollution in irrigation area with farmland as the core is becoming the main pollution source affecting the water quality of Aiyi River. Due to the large input of exogenous nutrients, the water exchange between rivers and lakes is weak, which makes the water body of Aiyi River tend to eutrophication gradually.

\section{Screening of comprehensive assessment indicators of aquatic ecosystem health in Aiyi River}

\section{Composition of candidate index system}

According to the results of factor analysis of water environment of Aiyi River, the main factors affecting water environment of Aiyi River are total phosphorus (TP), electrical conductivity (EC), ammonia nitrogen (NH3-N), total nitrogen (TN), 
permanganate index (CODMn), five-day biochemical oxygen demand (BOD5), suspended solids (SS), transparency (SD), chlorophyll a (Chl.a), so this paper determines these nine physical and chemical indicators as love. Candidate indicators of comprehensive evaluation index system of aquatic ecosystem health of Yihe River. Biological indicators include phytoplankton biomass, phytoplankton Shannon-Wiener index $\left(\mathrm{H}_{\mathrm{a}}^{\prime}\right)$, zooplankton Shannon-Wiener index $\left(\mathrm{H}^{\prime} \mathrm{b}\right)$, benthic animal ShannonWiener index $\left(\mathrm{H}^{\prime}{ }_{\mathrm{c}}\right)$, aquatic plant Shannon-Wiener index $\left(\mathrm{H}_{\mathrm{d}}\right)$, and sediment heterotrophic bacteria. At the same time, the comprehensive trophic status index $\left(\operatorname{TLI}_{(\Sigma)}\right)$ which can reflect the eutrophication characteristics of water body is selected. The above indicators together constitute the candidate indicators of the Aiyi River aquatic ecosystem health evaluation index system.

\section{Indicator screening method}

Principal component analysis was carried out for each index value of Aiyi River in March, May, July, September and November 2018. The factor load matrix was rotated by maximum variance orthogonal rotation method. The principal component was extracted according to $85 \%$ cumulative variance contribution rate. Then, the index with load value greater than 0.6 after rotation was selected as the index for evaluating the health attributes of Aiyi River (Boltovskoy, 2017).

\section{Determination of weight of evaluation index system}

The above selected indicators constitute an index system for evaluating the health attributes of Aiyi River, and then the principal component analysis is carried out. The eigenvalues of principal components, the contribution rate of variance and the load value of factor after rotation are obtained. According to the $85 \%$ cumulative variance contribution rate, four principal components are extracted. According to the corresponding eigenvalues and contribution rate of variance of the four principal components, the weight of the index can be calculated (David et al., 2017; Łuczyńska et al., 2018). The calculation formula is as follows:

$$
W_{i}=\sum_{j=1}^{k}\left|a_{i j}\right| \bullet E_{j}
$$

In the formula, $a_{i j}$ is the factor score coefficient (eigenvalue) of factor $i$ in the $j$ principal component, that is the contribution of factor $i$ to the $j$ principal component; $\mathrm{E}_{\mathrm{j}}$ is the contribution rate of the principal component to the variance; $\mathrm{W}_{\mathrm{i}}$ is the weight value of factor $i$. Then the weights of each evaluation factor can be obtained by normalizing the $\mathrm{W}_{\mathrm{i}}$.

\section{Determination of evaluation standard system}

Referring to "Standard for Surface Water Environmental Quality" (GB 3838-2002) (Gdara et al., 2018) and Hyun et al. (2017), Boltovskoy (2017) and Xiao-Yun et al. (2018), the evaluation standard system of Aquatic Ecosystem Health of Aiyi River was established, which was divided into five evaluation levels: very healthy, subhealthy, unhealthy and morbid (Table 1). 
Table 1. Classification of health assessment standard system

\begin{tabular}{|c|c|c|c|c|c|}
\hline Grade & Very healthy & Healthy & Sub-healthy & Unhealthy & Morbid \\
\hline Meaning & $\begin{array}{l}\text { The structure and } \\
\text { function of } \\
\text { aquatic } \\
\text { ecosystem are } \\
\text { complete and } \\
\text { balanced, the } \\
\text { flow of substance } \\
\text { and energy is } \\
\text { smooth, the } \\
\text { vitality and } \\
\text { resilience are } \\
\text { very strong, and } \\
\text { the health } \\
\text { condition is very } \\
\text { good }\end{array}$ & $\begin{array}{c}\text { Aquatic } \\
\text { ecosystems } \\
\text { maintain a } \\
\text { dynamic balance, } \\
\text { strong vitality, } \\
\text { reasonable } \\
\text { structure, } \\
\text { coordination, } \\
\text { strong resilience } \\
\text { and good health }\end{array}$ & $\begin{array}{l}\text { The structure of } \\
\text { aquatic } \\
\text { ecosystem has } \\
\text { changed, its } \\
\text { function has } \\
\text { declined, its } \\
\text { ecological } \\
\text { quality has } \\
\text { declined, its } \\
\text { vitality and } \\
\text { resilience are } \\
\text { general, and its } \\
\text { health status is } \\
\text { general }\end{array}$ & $\begin{array}{c}\text { Aquatic } \\
\text { ecosystems are } \\
\text { deteriorating } \\
\text { further, with } \\
\text { weak vitality, } \\
\text { inconsistent } \\
\text { structure, poor } \\
\text { resilience and } \\
\text { poor health }\end{array}$ & $\begin{array}{l}\text { Aquatic ecosystem } \\
\text { deteriorates } \\
\text { seriously, its } \\
\text { vitality is very } \\
\text { weak, its structure } \\
\text { is completely } \\
\text { unreasonable, its } \\
\text { resilience is very } \\
\text { poor, and its health } \\
\text { is very poor }\end{array}$ \\
\hline
\end{tabular}

\section{Health grade assessment of aquatic ecosystem in Aiyi River}

The health grade of Aiyi River was evaluated by grey correlation method. Taking health standard grading as comparison series, the measured values of each index are reference series, and the correlation degree between monthly and annual average values and health levels is calculated. The health level of water body in each period is judged by the magnitude of correlation degree (Boltovskoy, 2017; Fleming et al., 2002).

The evaluation steps are as follows (Boltovskoy, 2017; Fleming et al., 2002):

(1) Normalize the evaluation month and the index values of the evaluation criteria;

(2) Calculate the absolute difference between the normalized index value and the corresponding evaluation criteria of five evaluation grades $\left[\Delta_{\mathrm{ik}}(\mathrm{j})\right]$.

(3) Find out the minimum absolute difference $[\Delta \mathrm{min}]$ and the maximum absolute difference $[\Delta \max ]$ between all indexes and five evaluation grades.

(4) The correlation coefficients $\left[\varepsilon_{\mathrm{ik}}(\mathrm{j})\right]$ between the five-month and annual average values of each index and the corresponding evaluation criteria were calculated by taking the resolution coefficient $\mathrm{p}=0.5$.

$$
\varepsilon_{i k}(j)=\frac{\Delta \min +\rho \Delta \max }{\Delta_{i k}(j)+\rho \Delta \max }
$$

(5) According to the weight value of each index, the grey correlation degree $\left(\gamma_{\mathrm{ij}}\right)$ between the monthly and annual average and the five evaluation grades was calculated.

$$
\gamma_{i j}=W_{i} \varepsilon_{i k}(j)
$$

(6) According to the principle of maximum membership degree, the average health grade of 5 months and the whole year was evaluated. 


\section{Results}

\section{Selection of health assessment indicators for aquatic ecosystem of Aiyi River}

Four principal components were extracted according to $85 \%$ cumulative variance contribution rate (Table 2). Fifteen factors were selected according to the principle that the factor load value was greater than 0.6. However, the number of heterotrophic bacteria in sediment has not been reported as an indicator of aquatic ecosystem health assessment. The evaluation criteria are uncertain, so 14 factors such as SS, EC, COD $\mathrm{Mn}$, $\mathrm{BOD}_{5}, \mathrm{TN}, \mathrm{NH}_{3}-\mathrm{N}, \mathrm{TP}, \mathrm{Chl} . \mathrm{a}, \mathrm{TLI}_{(\Sigma)}$, phytoplankton biomass (D), phytoplankton $\mathrm{H}_{\mathrm{a}}$, zooplankton $\mathrm{H}_{\mathrm{b}}$, benthic animal $\mathrm{H}_{\mathrm{c}}$, and aquatic plant $\mathrm{H}_{\mathrm{d}}$, are identified as the indicators for the health evaluation of the Aquatic Ecosystem of Aiyi River (see Table 3).

\section{Composition and weight of health evaluation index system for aquatic ecosystem of Aiyi River}

Fourteen health assessment factors of Aquatic Ecosystem of Aiyi River, including SS, EC, COD $\mathrm{Mn}_{1}, \mathrm{BOD}_{5}, \mathrm{TN}, \mathrm{NH}_{3}-\mathrm{N}, \mathrm{TP}, \mathrm{Chl} . \mathrm{a}, \mathrm{TLI}_{(\Sigma)}$, phytoplankton biomass (D), phytoplankton $\mathrm{H}^{\prime}$, zooplankton $\mathrm{H}^{\prime}$, benthic animal $\mathrm{H}^{\prime}$, and aquatic plant $\mathrm{H}^{\prime}{ }_{\mathrm{d}}$ were screened and analyzed by principal component analysis. According to the analysis results (Table 4), the weights of each evaluation index were calculated, and the weights of 14 indicators were summed up. The normalized weights of each index can be obtained by dividing the weights of each index by the sum of weights (Table 5).

Table 2. Characteristic number and principal constituent contribution rate and the cumulative contribution rate

\begin{tabular}{c|c|c|c|c}
\hline Composition & F1 & F2 & F3 & F4 \\
\hline EC & 0.3813 & -0.0582 & 0.138 & 0.0336 \\
SD & 0.3115 & 0.0387 & 0.3545 & 0.0933 \\
SS & -0.1348 & 0.4475 & -0.1951 & -0.1855 \\
COD $_{\text {Mn }}$ & -0.2872 & -0.047 & -0.0525 & 0.3513 \\
BOD $_{5}$ & -0.2852 & -0.0468 & -0.0297 & 0.3371 \\
TN & 0.2368 & 0.3261 & 0.0771 & 0.3273 \\
NH$_{3}-\mathrm{N}$ & 0.1831 & 0.3704 & -0.0366 & 0.3359 \\
TP & 0.3009 & 0.0317 & -0.2581 & 0.3026 \\
Chl.a & -0.2917 & 0.1819 & -0.0505 & -0.3564 \\
TLI $(\Sigma)$ & -0.2067 & 0.3387 & -0.2682 & 0.1945 \\
Phytoplankton biomass & -0.114 & 0.0237 & 0.4751 & 0.1424 \\
Phytoplankton H'a & -0.263 & 0.2013 & 0.3765 & 0.0754 \\
Zooplankton H'b & 0.1178 & 0.1392 & 0.4363 & -0.2858 \\
Benthic animal H'c & -0.0916 & 0.4604 & 0.2166 & 0.0475 \\
Aquatic plant H'd & 0.1683 & 0.3406 & -0.161 & -0.3547 \\
Number of & 0.3673 & 0.0985 & -0.1857 & -0.0814 \\
heterotrophic bacteria & 6.1575 & 3.4362 & 2.9546 & 2.4977 \\
Characteristic value & 38.48 & 21.48 & 18.47 & 15.61 \\
Contribution rate\% & & 59.96 & 78.43 & 94.04 \\
Accumulated & 38.48 & & & \\
contribution rate\% & & &
\end{tabular}


Normalized weight values of SS, EC, $\mathrm{COD}_{\mathrm{Mn}}, \mathrm{BOD}_{5}, \mathrm{TN}, \mathrm{NH}_{3}-\mathrm{N}, \mathrm{TP}, \mathrm{Chl} . \mathrm{a}, \mathrm{TLI}_{(\Sigma)}$, phytoplankton biomass (D), phytoplankton $\mathrm{H}^{\prime}{ }_{\mathrm{a}}$, zooplankton $\mathrm{H}_{\mathrm{b}}$, benthic animal $\mathrm{H}_{\mathrm{c}}$, and aquatic plant $\mathrm{H}^{\prime}{ }_{\mathrm{d}}$ are respectively set as $0.0636,0.0724,0.0723,0.0711,0.0787$, 0.0764, 0.0716, 0.0756, 0.0771, 0.0790, 0.0571, 0.0599, 0.0717, 0.0736.

Table 3. Rotated factor matrix

\begin{tabular}{c|c|c|c|c}
\hline & $\mathbf{F 1}$ & $\mathbf{F 2}$ & $\mathbf{F 3}$ & $\mathbf{F 4}$ \\
\hline $\mathrm{EC}$ & $\mathbf{0 . 6 3 4 3}$ & 0.4997 & -0.5434 & -0.1365 \\
$\mathrm{SD}$ & 0.5750 & 0.5594 & -0.5047 & 0.3120 \\
$\mathrm{SS}$ & 0.0311 & 0.0506 & $\mathbf{0 . 9 9 6 9}$ & 0.0327 \\
$\mathrm{COD}_{\mathrm{Mn}}$ & $\mathbf{- 0 . 8 9 1 5}$ & -0.0718 & 0.0596 & 0.1687 \\
$\mathrm{BOD}_{5}$ & $\mathbf{- 0 . 8 6 3 1}$ & -0.0835 & 0.0504 & 0.2010 \\
$\mathrm{TN}$ & 0.1681 & $\mathbf{0 . 9 7 4 9}$ & 0.0504 & 0.1217 \\
$\mathrm{NH}_{3}-\mathrm{N}$ & 0.0354 & $\mathbf{0 . 9 5 2 6}$ & 0.2339 & 0.0163 \\
$\mathrm{TP}$ & 0.0703 & $\mathbf{0 . 7 5 4 5}$ & -0.2135 & -0.6053 \\
Chl.a & -0.0624 & -0.5945 & $\mathbf{0 . 7 4 2 8}$ & 0.2310 \\
TLI $(\Sigma)$ & -0.5630 & 0.2432 & $\mathbf{0 . 7 6 5 7}$ & -0.0414 \\
Phytoplankton biomass & -0.1349 & 0.0161 & -0.2341 & $\mathbf{0 . 8 5 2 2}$ \\
Phytoplankton H'a & -0.2967 & -0.0873 & 0.2603 & $\mathbf{0 . 9 1 3 8}$ \\
Zooplankton H'b & $\mathbf{0 . 7 3 0 4}$ & -0.0023 & -0.0459 & 0.6188 \\
Benthic animal H'c & 0.0278 & 0.3649 & 0.598 & $\mathbf{0 . 6 5 7 9}$ \\
Aquatic plant H'd & $\mathbf{0 . 6 9 7 3}$ & 0.1862 & 0.6199 & -0.242 \\
Number of & $\mathbf{0 . 6 4 6 6}$ & 0.5101 & -0.0367 & -0.5503 \\
heterotrophic bacteria & & \multicolumn{3}{|c}{} \\
\hline
\end{tabular}

Table 4. Characteristic number and principal constituent contribution rate and the cumulative contribution rate

\begin{tabular}{c|c|c|c|c}
\hline Composition & F1 & F2 & F3 & F4 \\
\hline EC & -0.4211 & 0.0178 & -0.1168 & 0.1645 \\
SD & 0.1993 & 0.4184 & 0.0275 & -0.3055 \\
SS & 0.3177 & -0.1062 & 0.3107 & 0.1964 \\
COD $_{\text {Mn }}$ & 0.3178 & -0.1066 & 0.2836 & 0.2038 \\
BOD $_{5}$ & -0.2185 & 0.3794 & 0.1362 & 0.3196 \\
TN & -0.1622 & 0.416 & 0.2325 & 0.2324 \\
$\mathrm{NH}_{3}-\mathrm{N}$ & -0.3506 & 0.105 & 0.3736 & 0.0473 \\
TP & 0.3439 & 0.1183 & -0.1813 & -0.3296 \\
Chl.a & 0.2548 & 0.3043 & 0.3504 & -0.0852 \\
TLI $(\Sigma)$ & 0.3423 & 0.141 & -0.2143 & 0.3269 \\
Phytoplankton biomass & 0.1502 & -0.0006 & -0.2572 & 0.468 \\
Phytoplankton H'a & -0.0796 & 0.1486 & -0.5403 & 0.137 \\
Zooplankton H'b & 0.1819 & 0.4339 & -0.1305 & 0.1926 \\
Benthic animal H'c & -0.1474 & 0.3703 & -0.1405 & -0.3787 \\
Aquatic plant H'd & 4.7865 & 3.3635 & 2.5934 & 2.3328 \\
Number of & 34.19 & 24.03 & 18.52 & 16.66 \\
heterotrophic bacteria & 34.19 & 58.21 & 76.74 & 93.40 \\
Characteristic value & & &
\end{tabular}


Table 5. The weight and the normalized weight of evaluation indicators

\begin{tabular}{|c|c|c|c|c|c|c|c|c|c|c|}
\hline & \multicolumn{4}{|c|}{$\begin{array}{c}\text { Principal component eigenvalues of } \\
\text { evaluation indicators }\end{array}$} & \multicolumn{4}{|c|}{$\begin{array}{l}\text { Variance contribution rate of } \\
\text { each principal component }\end{array}$} & \multirow{2}{*}{ Weight } & \multirow{2}{*}{$\begin{array}{c}\text { Normalized } \\
\text { weight }\end{array}$} \\
\hline & F1 & F2 & F3 & F4 & F1 & F2 & F3 & F4 & & \\
\hline SS & -0.4211 & 0.0178 & -0.1168 & 0.1645 & 34.19 & 24.03 & 18.52 & 16.66 & 0.1973 & 0.0636 \\
\hline $\mathrm{EC}$ & 0.1993 & 0.4184 & 0.0275 & -0.3055 & 34.19 & 24.03 & 18.52 & 16.66 & 0.2247 & 0.0724 \\
\hline $\mathrm{COD}_{\mathrm{Mn}}$ & 0.3177 & -0.1062 & 0.3107 & 0.1964 & 34.19 & 24.03 & 18.52 & 16.66 & 0.2244 & 0.0723 \\
\hline $\mathrm{BOD}_{5}$ & 0.3178 & -0.1066 & 0.2836 & 0.2038 & 34.19 & 24.03 & 18.52 & 16.66 & 0.2208 & 0.0711 \\
\hline $\mathrm{TN}$ & -0.2185 & 0.3794 & 0.1362 & 0.3196 & 34.19 & 24.03 & 18.52 & 16.66 & 0.2443 & 0.0787 \\
\hline $\mathrm{NH}_{4}-\mathrm{N}$ & -0.1622 & 0.416 & 0.2325 & 0.2324 & 34.19 & 24.03 & 18.52 & 16.66 & 0.2372 & 0.0764 \\
\hline $\mathrm{TP}$ & -0.3506 & 0.105 & 0.3736 & 0.0473 & 34.19 & 24.03 & 18.52 & 16.66 & 0.2222 & 0.0716 \\
\hline Chl.a & 0.3439 & 0.1183 & -0.1813 & -0.3296 & 34.19 & 24.03 & 18.52 & 16.66 & 0.2345 & 0.0756 \\
\hline $\operatorname{TLI}_{(\Sigma)}$ & 0.2548 & 0.3043 & 0.3504 & -0.0852 & 34.19 & 24.03 & 18.52 & 16.66 & 0.2393 & 0.0771 \\
\hline Phytoplankton biomass D & 0.3423 & 0.141 & -0.2143 & 0.3269 & 34.19 & 24.03 & 18.52 & 16.66 & 0.2451 & 0.079 \\
\hline Phytoplankton H'a & 0.1502 & -0.0006 & -0.2572 & 0.468 & 34.19 & 24.03 & 18.52 & 16.66 & 0.1771 & 0.0571 \\
\hline Zooplankton H'b & -0.0796 & 0.1486 & -0.5403 & 0.137 & 34.19 & 24.03 & 18.52 & 16.66 & 0.1858 & 0.0599 \\
\hline Benthic animal H'c & 0.1819 & 0.4339 & -0.1305 & 0.1926 & 34.19 & 24.03 & 18.52 & 16.66 & 0.2227 & 0.0717 \\
\hline Aquatic plant $\mathrm{H}^{\prime} \mathrm{d}$ & -0.1474 & 0.3703 & -0.1405 & -0.3787 & 34.19 & 24.03 & 18.52 & 16.66 & 0.2285 & 0.0736 \\
\hline
\end{tabular}

\section{Standard system for health assessment of aquatic ecosystem in Aiyi River}

The classification standard of physical and chemical indexes of water body is based on "Surface Water Environmental Quality Standard" (GB 3838-2002). Five grades of very healthy, healthy, sub-healthy, unhealthy and sick correspond to I, II, III, IV and V water standards respectively. The classification standard of suspended solids (SS) and conductivity (EC) is determined by reference (Boltovskoy, 2017).

According to the relevant provisions of the "Evaluation Method of Eutrophication of Lakes (Reservoirs) and Technical Regulations for Classification" (Yang et al., 2019; Ochoa-Rivero et al., 2017; Sorokovikova et al., 2017), the comprehensive nutritional status index $\mathrm{N}=50$ is medium nutrition, which is regarded as sub-health level, $\mathrm{N}<30$ is poor nutrition, which is very healthy level, $\mathrm{N}>70$ is severe eutrophication, which is morbid level, 30-50 is health level, 60-70 is unhealthy level.

The Shannon-Wiener diversity index (H') of phytoplankton, zooplankton, benthic and aquatic plants reflects the diversity of community species. The value of $H^{\prime}(0-1)$ is eutrophic, 1-3 is mesotrophic and more than 3 is poor nutrition (Kozlov et al., 2017). Accordingly, 2-3 is considered as sub-health grade, 4 is considered as very health grade, $H^{\prime}(1)$ is considered as morbid grade, 3-4 is health grade, 1-2 is unhealthy grade.

According to the relevant evaluation criteria of lake eutrophication in China (Suzuki et al., 2018), phytoplankton biomass $<3 \mathrm{mg} \mathrm{L}^{-1}$ is a poor nutrition type, which is regarded as very healthy and healthy grade. $3-5 \mathrm{mg} \mathrm{L}^{-1}$ is a middle nutrition type, which is regarded as sub-health grade, 5-10 $\mathrm{mg} \mathrm{L}^{-1}$ as eutrophic type, which is unhealthy grade, and $>10 \mathrm{mg} \mathrm{L}^{-1}$ as extremely eutrophic type, which is a morbid grade. 
The health assessment standard system of the Aquatic Ecosystem of Aiyi River is shown in Table 6.

Table 6. Health assessment standard system of Aiyi River aquatic ecosystem

\begin{tabular}{c|c|c|c|c|c}
\hline \multirow{2}{*}{ Index } & \multicolumn{5}{|c}{ Health grade } \\
\cline { 2 - 6 } & Very healthy & Healthy & Sub-healthy & Unhealthy & Morbid \\
\hline $\mathrm{EC}\left(\mu \mathrm{S} \cdot \mathrm{cm}^{-1}\right)$ & $\leq 200$ & $(200,300]$ & $(300,400]$ & $(400,500]$ & $(500,600]$ \\
$\mathrm{SS}\left(\mathrm{mg} \cdot \mathrm{L}^{-1}\right)$ & $\leq 96$ & $(96,172]$ & $(172,364]$ & $(364,820]$ & $(820,1640]$ \\
$\mathrm{COD}_{\mathrm{Mn}}\left(\mathrm{mg} \cdot \mathrm{L}^{-1}\right)$ & $\leq 2$ & $(2,4]$ & $(4,6]$ & $(6,10]$ & $(10,15]$ \\
$\left.\mathrm{BOD} 5 \mathrm{mg} \cdot \mathrm{L}^{-1}\right)$ & $\leq 3.0$ & $\leq 3.0$ & $(3.0,4.0]$ & $(4.0,6.0]$ & $(6.0,10.0]$ \\
$\mathrm{TN}\left(\mathrm{mg} \cdot \mathrm{L}^{-1}\right)$ & $\leq 0.2$ & $(0.2,0.5]$ & $(0.5,1.0]$ & $(1.0,1.5]$ & $(1.5,2.0]$ \\
$\mathrm{NH}_{3}-\mathrm{N}\left(\mathrm{mg} \cdot \mathrm{L}^{-1}\right)$ & $\leq 0.15$ & $(0.15,0.50]$ & $(0.50,1.0]$ & $(1.0,1.5]$ & $(1.5,2.0]$ \\
$\mathrm{TP}\left(\mathrm{mg} \cdot \mathrm{L}^{-1}\right)$ & $\leq 0.02$ & $(0.02,0.10]$ & $(0.10,0.20]$ & $(0.20,0.30]$ & $(0.30,0.40]$ \\
$\mathrm{Chl} \cdot \mathrm{a}\left(\mu \mathrm{g} \cdot \mathrm{L}^{-1}\right)$ & $\leq 1.0$ & $(1.0,2.0]$ & $(2.0,4.0]$ & $(4.0,10.0]$ & $(10.0,26.0]$ \\
$\mathrm{TLI}(\Sigma)$ & $\leq 30$ & $(30,50]$ & $(50,60]$ & $(60,70]$ & $>70$ \\
Phytoplankton biomass D & $\leq 3.0$ & $\leq 3.0$ & {$[3.0,5.0)$} & {$[5.0,10.0)$} & $\geq 10.0$ \\
Phytoplankton H'a & $\geq 4.0$ & {$[3.0,4.0)$} & {$[2.0,3.0)$} & {$[1.0,2.0)$} & $\leq 1.0$ \\
Zooplankton H'b & $\geq 3.0$ & {$[2.0,3.0)$} & {$[1.0,2.0)$} & {$[0.5,1.0)$} & $\leq 0.5$ \\
Benthic animal H'c & $\geq 3.0$ & {$[2.0,3.0)$} & {$[1.0,2.0)$} & {$[0.5,1.0)$} & $\leq 0.5$ \\
Aquatic plant H'd & $\geq 3.0$ & {$[2.0,3.0)$} & {$[1.0,2.0)$} & {$[0.5,1.0)$} & $\leq 0.5$ \\
\hline
\end{tabular}

\section{Health grade assessment of aquatic ecosystem in Aiyi River}

Taking the annual average index of Aiyi River as an example, the correlation degree between each evaluation index and evaluation standard grade was calculated (Table 7). The results of the difference calculation are shown in Table 8 , and the correlation coefficient and correlation degree are shown in Tables 9 and 10.

Table 7. The measured values of evaluation indicators in AiYi River

\begin{tabular}{c|c|c|c|c|c|c}
\hline & March & May & July & September & November & Avg. \\
\hline EC(us'cm-1 $)$ & $\mathbf{1 1 1 6 . 6 7}$ & $\mathbf{1 0 5 6 . 6 7}$ & $\mathbf{9 9 1 . 6 7}$ & $\mathbf{9 2 8 . 3 3}$ & $\mathbf{9 8 1 . 6 7}$ & $\mathbf{1 0 1 5 . 0 0}$ \\
\hline $\mathrm{SS}\left(\mathrm{mg} \cdot \mathrm{L}^{-1}\right)$ & 23.24 & 34.62 & 42.42 & 25.28 & 25.24 & 30.16 \\
$\left.\mathrm{COD} \mathrm{Mn}_{\mathrm{mng}} \cdot \mathrm{L}^{-1}\right)$ & 6.03 & 6.13 & 9.98 & 8.93 & 5.75 & 7.36 \\
$\mathrm{BOD}\left(\mathrm{mg} \cdot \mathrm{L}^{-1}\right)$ & 2.98 & 3.17 & 5.18 & 5.78 & 3.01 & 4.02 \\
$\mathrm{TN}\left(\mathrm{mg} \cdot \mathrm{L}^{-1}\right)$ & 1.835 & 2.302 & 3.080 & 1.247 & 3.063 & 2.305 \\
$\mathrm{NH}{ }_{3}-\mathrm{N}\left(\mathrm{mg} \cdot \mathrm{L}^{-1}\right)$ & 0.634 & 0.745 & 0.989 & 0.866 & 0.440 & 0.735 \\
$\mathrm{TP}\left(\mathrm{mg} \cdot \mathrm{L}^{-1}\right)$ & 0.082 & 0.050 & 0.094 & 0.118 & 0.064 & 0.082 \\
$\mathrm{Chl.a}\left(\mathrm{ug} \cdot \mathrm{L}^{-1}\right)$ & 10.51 & 10.49 & 15.10 & 13.58 & 11.62 & 12.26 \\
$T L I_{(\Sigma)}$ & 53.94 & 54.26 & 62.4 & 57.13 & 54.54 & 56.45 \\
Phytoplankton biomass $\left(\mathrm{mg} \cdot \mathrm{L}^{-1}\right)$ & 4.52 & 6.58 & 6.02 & 5.17 & 3.43 & 5.15 \\
Phytoplankton H'a & 3.469 & 3.464 & 3.184 & 2.993 & 3.558 & 3.334 \\
Zooplankton H'b & 2.648 & 2.728 & 3.094 & 2.965 & 3.356 & 2.958 \\
Benthic animal H'c & 2.043 & 2.004 & 2.284 & 2.175 & 1.575 & 2.016 \\
Aquatic plant $\mathrm{H}^{\prime} \mathrm{d}$ & 2.441 & 2.002 & 2.118 & 2.294 & 2.410 & 2.424 \\
\hline
\end{tabular}


Table 8. D-value of average index and evaluation criterion

\begin{tabular}{c|c|c|c|c|c|c|c}
\hline & \multicolumn{7}{|c}{ Difference value } \\
\cline { 2 - 8 } & $\begin{array}{c}\text { Very } \\
\text { healthy }\end{array}$ & Healthy & $\begin{array}{c}\text { Sub- } \\
\text { healthy }\end{array}$ & Unhealthy & Morbid & Max & Min \\
\hline $\mathrm{EC}\left(\mu \mathrm{S} \cdot \mathrm{cm}^{-1}\right)$ & 815 & 715 & 615 & 515 & 415 & 815 & 415 \\
$\mathrm{SS}\left(\mathrm{mg} \cdot \mathrm{L}^{-1}\right)$ & 65.84 & 141.84 & 333.84 & 789.84 & 1609.84 & 1609.84 & 65.84 \\
$\left.\mathrm{COD} \mathrm{Mn}_{\mathrm{m}} \cdot \mathrm{m} \cdot \mathrm{L}^{-1}\right)$ & 5.36 & 3.36 & 1.36 & 2.64 & 7.64 & 7.64 & 1.36 \\
$\mathrm{BOD}\left(\mathrm{mg} \cdot \mathrm{L}^{-1}\right)$ & 1.02 & 1.02 & 0.02 & 1.98 & 5.98 & 5.98 & 0.02 \\
$\mathrm{TN}\left(\mathrm{mg} \cdot \mathrm{L}^{-1}\right)$ & 2.105 & 1.805 & 1.305 & 0.805 & 0.305 & 2.105 & 0.305 \\
$\mathrm{NH}_{3}-\mathrm{N}\left(\mathrm{mg} \cdot \mathrm{L}^{-1}\right)$ & 0.585 & 0.235 & 0.265 & 0.765 & 1.265 & 1.265 & 0.235 \\
$\mathrm{TP}\left(\mathrm{mg} \cdot \mathrm{L}^{-1}\right)$ & 0.062 & 0.018 & 0.118 & 0.218 & 0.318 & 0.318 & 0.018 \\
$\mathrm{Chl} \cdot \mathrm{a}\left(\mu \mathrm{g} \cdot \mathrm{L}^{-1}\right)$ & 11.26 & 10.26 & 8.26 & 2.26 & 7.74 & 11.26 & 2.26 \\
$T L I_{(\Sigma)}$ & 26.45 & 6.45 & 3.55 & 13.55 & 43.55 & 43.55 & 3.55 \\
Phytoplankton biomass $\left(\mathrm{mg} \cdot \mathrm{L}^{-1}\right)$ & 1.15 & 2.15 & 0.15 & 4.85 & 14.85 & 14.85 & 0.15 \\
Phytoplankton H'a & 0.666 & 0.334 & 1.334 & 2.334 & 3.334 & 3.334 & 0.334 \\
Zooplankton H'b & 1.042 & 0.042 & 0.958 & 1.958 & 2.958 & 2.958 & 0.042 \\
Benthic animal H'c & 1.984 & 0.984 & 0.016 & 1.016 & 2.016 & 2.016 & 0.016 \\
Aquatic plant H'd & 1.576 & 0.576 & 0.424 & 1.424 & 2.424 & 2.424 & 0.424 \\
\hline
\end{tabular}

Table 9. The grey relational coefficient of average index and evaluation criterion $\varepsilon_{i k}(j)$

\begin{tabular}{c|c|c|c|c|c}
\hline & \multicolumn{5}{|c}{ Relational coefficient } \\
\cline { 2 - 6 } & Very healthy & Healthy & Sub-healthy & Unhealthy & Morbid \\
\hline $\mathrm{EC}\left(\mu \mathrm{S} \cdot \mathrm{cm}^{-1}\right)$ & 0.6728 & 0.7327 & 0.8044 & 0.8916 & 1.0000 \\
$\mathrm{SS}\left(\mathrm{mg} \cdot \mathrm{L}^{-1}\right)$ & 1.0000 & 0.9197 & 0.7647 & 0.546 & 0.3606 \\
$\mathrm{COD}_{\mathrm{Mn}}\left(\mathrm{mg} \cdot \mathrm{L}^{-1}\right)$ & 0.5643 & 0.7214 & 1.0000 & 0.8019 & 0.452 \\
$\mathrm{BOD} 5\left(\mathrm{mg} \cdot \mathrm{L}^{-1}\right)$ & 0.7506 & 0.7506 & 1.0000 & 0.6056 & 0.3356 \\
$\mathrm{TN}\left(\mathrm{mg} \cdot \mathrm{L}^{-1}\right)$ & 0.4299 & 0.4751 & 0.5758 & 0.7308 & 1.0000 \\
$\mathrm{NH}_{3}-\mathrm{N}\left(\mathrm{mg} \cdot \mathrm{L}^{-1}\right)$ & 0.7125 & 1.0000 & 0.9666 & 0.6208 & 0.4572 \\
$\mathrm{TP}\left(\mathrm{mg} \cdot \mathrm{L}^{-1}\right)$ & 0.8009 & 1.0000 & 0.639 & 0.4695 & 0.3711 \\
$\mathrm{Chl} \cdot \mathrm{a}\left(\mu \mathrm{g} \cdot \mathrm{L}^{-1}\right)$ & 0.4671 & 0.4965 & 0.568 & 1.0000 & 0.5901 \\
$T L I_{(\Sigma)}$ & 0.5251 & 0.8973 & 1.0000 & 0.7169 & 0.3877 \\
Phytoplankton biomass $\left(\mathrm{mg} \cdot \mathrm{L}^{-1}\right)$ & 0.8834 & 0.7911 & 1.0000 & 0.6171 & 0.3401 \\
Phytoplankton H'a & 0.8577 & 1.0000 & 0.6668 & 0.5001 & 0.4001 \\
Zooplankton H'b & 0.6033 & 1.0000 & 0.6241 & 0.4425 & 0.3428 \\
Benthic animal H'c & 0.3422 & 0.5141 & 1.0000 & 0.5059 & 0.3386 \\
Aquatic plant $\mathrm{H}^{\prime} \mathrm{d}$ & 0.5868 & 0.915 & 1.0000 & 0.6206 & 0.4499 \\
\hline
\end{tabular}

The correlation between each month and the grade of evaluation criteria is calculated in Table 11. From Table 11 we can see that the evaluation results of the Aiyi River in March and November are healthy, the evaluation results in May, July and September are sub-healthy, and the overall evaluation of the health status of the Aiyi River aquatic ecosystem is sub-healthy. 
Table 10. Grey relational degree of average index and evaluation criterion

\begin{tabular}{c|c|c|c|c|c|c}
\hline & Weight & Very healthy & Healthy & Sub-healthy & Unhealthy & Morbid \\
\hline $\mathrm{EC}\left(\mu \mathrm{S} \cdot \mathrm{cm}^{-1}\right)$ & 0.0636 & 0.0428 & 0.0466 & 0.0512 & 0.0567 & 0.0636 \\
$\mathrm{SS}\left(\mathrm{mg} \cdot \mathrm{L}^{-1}\right)$ & 0.0724 & 0.0724 & 0.0666 & 0.0554 & 0.0395 & 0.0261 \\
$\mathrm{COD}_{\mathrm{Mn}}\left(\mathrm{mg} \cdot \mathrm{L}^{-1}\right)$ & 0.0723 & 0.0408 & 0.0522 & 0.0723 & 0.058 & 0.0327 \\
$\mathrm{BOD}\left(\mathrm{mg} \cdot \mathrm{L}^{-1}\right)$ & 0.0711 & 0.0534 & 0.0534 & 0.0711 & 0.0431 & 0.0239 \\
$\mathrm{TN}\left(\mathrm{mg} \cdot \mathrm{L}^{-1}\right)$ & 0.0787 & 0.0338 & 0.0374 & 0.0453 & 0.0575 & 0.0787 \\
$\mathrm{NH}_{3}-\mathrm{N}\left(\mathrm{mg} \cdot \mathrm{L}^{-1}\right)$ & 0.0764 & 0.0544 & 0.0764 & 0.0738 & 0.0474 & 0.0349 \\
$\mathrm{TP}\left(\mathrm{mg} \cdot \mathrm{L}^{-1}\right)$ & 0.0716 & 0.0573 & 0.0716 & 0.0458 & 0.0336 & 0.0266 \\
$\mathrm{Chl} \cdot \mathrm{a}\left(\mu \mathrm{g} \cdot \mathrm{L}^{-1}\right)$ & 0.0756 & 0.0353 & 0.0375 & 0.0429 & 0.0756 & 0.0446 \\
$T L I_{(\Sigma)}$ & 0.0771 & 0.0405 & 0.0692 & 0.0771 & 0.0553 & 0.0299 \\
Phytoplankton biomass $\left(\mathrm{mg} \cdot \mathrm{L}^{-1}\right)$ & 0.079 & 0.0698 & 0.0625 & 0.079 & 0.0488 & 0.0269 \\
Phytoplankton H'a & 0.0571 & 0.049 & 0.0571 & 0.0381 & 0.0286 & 0.0228 \\
Zooplankton H'b & 0.0599 & 0.0361 & 0.0599 & 0.0374 & 0.0265 & 0.0205 \\
Benthic animal H'c & 0.0717 & 0.0245 & 0.0369 & 0.0717 & 0.0363 & 0.0243 \\
Aquatic plant H'd & 0.0736 & 0.0432 & 0.0673 & 0.0736 & 0.0457 & 0.0331 \\
Correlation degree & & 0.6534 & 0.7945 & 0.8346 & 0.6525 & 0.4886 \\
\hline
\end{tabular}

Table 11. Calculation of grey relational degree

\begin{tabular}{c|c|c|c|c|c|c}
\hline Site & Very healthy & Healthy & Sub-healthy & Unhealthy & Morbid & Result \\
\hline March & 0.6743 & 0.8219 & 0.8093 & 0.64 & 0.4675 & Healthy \\
May & 0.6627 & 0.7751 & 0.8242 & 0.6461 & 0.4688 & Sub-healthy \\
July & 0.5995 & 0.7294 & 0.8078 & 0.7049 & 0.5171 & Sub-healthy \\
September & 0.5854 & 0.7521 & 0.8249 & 0.7102 & 0.4692 & Sub-healthy \\
November & 0.7428 & 0.8521 & 0.7944 & 0.6583 & 0.4939 & Healthy \\
Comprehensive & 0.6534 & 0.7945 & 0.8346 & 0.6525 & 0.4886 & Sub-healthy \\
\hline
\end{tabular}

\section{Conclusion}

\section{Health evaluation index of aquatic ecosystem in Aiyi River}

The evaluation index of Aquatic Ecosystem Health of Aiyi River is mainly composed of four parts: The first part includes the factors of phytoplankton biomass (D), phytoplankton $\mathrm{H}_{\mathrm{a}}$, zooplankton $\mathrm{H}_{\mathrm{b}}$, benthic animal $\mathrm{H}_{\mathrm{c}}{ }_{\mathrm{c}}$ and aquatic plant $\mathrm{H}_{\mathrm{d}}$ which are the comprehensive reflection of the diversity and integrity of aquatic ecosystem structure, and the most important part of the evaluation index of aquatic ecosystem health. The second part includes SS, EC, Chl.a and $\operatorname{TLI}_{(\Sigma)}$ which mainly describe the content of suspended solids and dissolved salts in water. The third part includes $\mathrm{TN}, \mathrm{NH}_{3}-\mathrm{N}$ and $\mathrm{TP}$, which mainly describe the composition and content of nitrogen and phosphorus nutrients in water. The fourth part includes $\mathrm{COD}_{\mathrm{Mn}}$ and $\mathrm{BOD}_{5}$ which mainly describe the content of organic matter in water. The above indicators cover the main water quality factors such as suspended matter, dissolved salts, nutrients and organic matter, which can fully reflect the water environment quality of the Aiyi River. Biological indicators include phytoplankton biomass (D), phytoplankton $\mathrm{H}_{\mathrm{a}}$, zooplankton $\mathrm{H}_{\mathrm{b}}$, benthic animal $\mathrm{H}_{\mathrm{c}}$, and aquatic plant $\mathrm{H}_{\mathrm{d}}$, which are comprehensive reflections of the structural diversity of aquatic ecosystems. In the process of research, 
due to the lack of microbial research results, microbial indicators can not be included in the evaluation index system, and further in-depth and systematic research is needed to further improve the theory and methods of aquatic ecosystem health assessment.

\section{Health status of aquatic ecosystem in Aiyi River}

The contents of S S, CODMn, BOD5, NH3-N, TN and TP in Aiyi River in May, July and September were significantly higher than those in March and November, indicating that the contents of nutrients and organic matter in the water body of Aiyi River were higher in May, July and September, and the pollution degree of the water body was higher. The reason was that a large amount of organic matter and nutrients entered the water body due to the large amount of water recharge in the three periods. At the same time, under the high nutrient and high temperature environment, cyanobacteria multiply in large quantities, forming dominant species, inhibiting the growth of other phytoplankton species, decreasing phytoplankton diversity, increasing chlorophyll a content, phytoplankton density and biomass, resulting in serious impacts on the structure of the aquatic ecosystem and functional degradation of the Aiyi River, so the health status in May, July and September is worse than that in March and November. To be poor, in a sub-health state. $\mathrm{TLI}_{(\Sigma)}$ indicates the degree of eutrophication of the water body. The $\operatorname{TLI}_{(\Sigma)}$ in July and September of the Aiyi River is higher than that in March and November. It also shows that the water body of the Aiyi River has been moderately polluted, in a state of medium eutrophication, and its health has declined. In addition, the community structure of aquatic plants and benthic fauna in Aiyi River is not balanced and the diversity is low. The natural succession rate of aquatic plants and benthic fauna in Aiyi River can not adapt to the pressures of exogenous pollutants, and the inadequate ability to degrade nitrogen, phosphorus nutrients and organic matter is also an important reason for the sub-health of the aquatic ecosystem in Aiyi River.

The water source of Aiyi River is mainly farmland recession with high nutrient and organic matter content, which is the main cause of water pollution of Aiyi River. The aquatic ecosystem is greatly affected by external stress factors, resulting in sub-health of the overall health of the aquatic ecosystem of Aiyi River. The comprehensive evaluation results also reflect the water environment characteristics of the aquatic ecosystem of Aiyi River comprehensively. According to the results of health assessment of aquatic ecosystem, the prevention and control of water pollution in Aiyi River should mainly focus on reducing the content of exogenous nutrients and organic matter, rebuilding and restoring aquatic ecosystem, ensuring its structural integrity and functional stability.

The health status of river aquatic ecosystem varies greatly in different time and space scales. Based on the actual monitoring data in 2018, the health status of Aiyi River aquatic ecosystem is preliminarily evaluated. In the future, the characteristics of aquatic plant community, the characteristics of algae community and its diversity index, biological integrity index and comprehensive quality evaluation index of habitat need to be strengthened. In order to further improve the theory and method of river aquatic ecosystem health assessment, we should strengthen the observation of long time series aquatic ecosystem in different water periods.

Acknowledgements. This project is supported by "Major Innovation Projects for Building First-class Universities in China’s Western Region” (ZKZD2017002). 


\section{REFERENCES}

[1] Boltovskoy, D. (2017): Traits and impacts of invasive species: myths and evidences from the perspective of introduced freshwater mussels. - Aquatic Ecosystem Health \& Management. DOI: 10.1080/14634988.2017.1397483.

[2] David, B., Avinash, K., Warish, A., Stephen, C., Paul, M., Christopher, S. et al. (2017): A community multi-omics approach towards the assessment of surface water quality in an urban river system. - International Journal of Environmental Research and Public Health 14(3): 303-310.

[3] Du, Y., Ye, J., Wu, L., Yang, C., Wang, L., Hu, X. (2017): Physiological effects and toxin release in microcystis aeruginosa and microcystis viridis exposed to herbicide fenoxaprop-p-ethyl. - Environmental Science and Pollution Research 24(8): 7752-7763.

[4] Fleming, R. A., Barclay, H. J., Candau, J. N. (2002): Scaling-up an autoregressive timeseries model (of spruce budworm population dynamics) changes its qualitative behaviour. - Ecological Modelling 149(1-2): 127-142.

[5] Gdara, I., Zrafi, I., Balducci, C., Cecinato, A., Ghrabi, A. (2018): Seasonal occurrence, source evaluation and ecological risk assessment of polycyclic aromatic hydrocarbons in industrial and agricultural effluents discharged in Wadi el Bey (Tunisia). - Environ Geochem Health 20: 1-19.

[6] Hossain, M., Stewart, T. J., Arhonditsis, G. B., Van Oevelen, D., Minns, C. K., Koops, M. A. (2017): Uncertainty assessment of trophic flows in hamilton harbour: a linear inverse modelling analysis. - Aquatic Ecosystem Health \& Management 20(3): 265-277.

[7] Hyun, B., Baek, S. H., Shin, K., Choi, K. H. (2017): Assessment of phytoplankton invasion risks in the ballast water of international ships in different growth conditions. Aquatic Ecosystem Health \& Management. https://doi.org/10.1080/14634988. 2017.1406273.

[8] Islam, M. R., Das, N. G., Barua, P., Hossain, M. B., Venkatramanan, S., Chung, S. Y. (2017): Environmental assessment of water and soil contamination in rajakhali canal of karnaphuli river (bangladesh) impacted by anthropogenic influences: a preliminary case study. - Applied Water Science 7(2): 997-1010.

[9] Janssens, L., Lin, O. D. B., Stoks, R. (2017): Stoichiometric responses to an agricultural pesticide are modified by predator cues. - Environmental Science \& Technology 51(1): 581-588.

[10] Kozlov, D. V., Nasonov, A. N., Tsvetkov, I. V., Zhogin, I. M. (2017): Multifractal principles of aquatic ecosystem development control by algacenosis correction. - Water Resources 44(2): 259-266.

[11] Łuczyńska, J., Paszczyk, B., Łuczyński, M. J. (2018): Fish as a bioindicator of heavy metals pollution in aquatic ecosystem of Pluszne Lake, Poland, and risk assessment for consumer's health. - Ecotoxicology \& Environmental Safety 153: 60-67.

[12] Maloney, E. M. (2019): How do we take the pulse of an aquatic ecosystem? current and historical approaches to measuring ecosystem integrity. - Environmental Toxicology and Chemistry 38(2).

[13] Milani, D., Grapentine, L., Burniston, D. A., Graham, M., Marvin, C. (2017): Trends in sediment quality in hamilton harbour, lake ontario. - Aquatic Ecosystem Health \& Management 20(3): 295-307.

[14] Obolewski, K., Glińska-Lewczuk, K., Bąkowska, M. (2018): From isolation to connectivity: the effect of floodplain lake restoration on sediments as habitats for macroinvertebrate communities. - Aquatic Sciences 80(1): 4.

[15] Ochoa-Rivero, J. M., Reyes-Fierro, A. V., Peralta-Pérez, M. D. R., Zavala-Díaz de la Serna, F. J. et al. (2017): Levels and distribution of pollutants in the waters of an aquatic ecosystem in northern Mexico. - International Journal of Environmental Research and Public Health 14(5): 456-465. 
[16] Richman, L., Milani, D., Marvin, C. (2017): Trends in suspended sediment quality in the upper st. clair river: assessment of large-scale remediation of contaminated sediments in a dynamic riverine environment. - Aquatic Ecosystem Health \& Management 21(1): 93106.

[17] Rongrong, W., Guishan, Y., Xue, D., Yanhui, Z., Bing, L. I. (2018): Water security-based hydrological regime assessment method for lakes with extreme seasonal water level fluctuations: a case study of Poyang Lake, China. - Chinese Geographical Science 3: 114.

[18] Sorokovikova, L. M., Sinyukovich, V. N., Tomberg, I. V. (2017): The status of the aquatic ecosystem of the selenga river delta under long-duration low-water conditions. Geography and Natural Resources 38(1): 60-67.

[19] Suzuki, K., Watanabe, S., Yuasa, Y., Yamashita, Y., Arai, H., Tanaka, H. et al. (2018): Adiocesium dynamics in the aquatic ecosystem of Lake Onuma on MT. AKAGI following the Fukushima Dai-Ichi nuclear power plant accident. - Science of the Total Environment 622-623: 1153-1164.

[20] Xiao-Yun, G. U., Zong-Xue, X. U., Lin-Fei, L., Xu-Wang, Y., Mi, W. (2018): Health assessment of the stream ecosystem in the North Canal River basin, Beijing, China. Environmental Science 39(6): 2576.

[21] Yang, J., Wang, F., Lv, J., Liu, Q., Feng, J. (2019): Responses of freshwater algal cell density to hydrochemical variables in an urban aquatic ecosystem, northern China. Environmental Monitoring and Assessment 191(1).

[22] Yu, C., Chen, S. S., Zhang, L., Gao, Q., Wang, Z., Shen, Q. (2017): Changes in water quality of the rivers discharging into Lake Tanganyika in Bujumbura, Burundi. - Aquatic Ecosystem Health \& Management. https://doi.org/10.1080/14634988.2017.1394772. 